Published as: Delafontaine, M., Neutens, T. \& Van de Weghe, N. (2011), Modelling potential movement in constrained travel environments using rough space-time prisms. International Journal of Geographical Information Science, vol. 25 (9), 1389-1411.

\title{
Modelling potential movement in constrained travel environments using rough space-time prisms
}

\author{
Matthias Delafontaine, Tijs Neutens and Nico Van de Weghe \\ \{Matthias.Delafontaine, Tijs.Neutens, Nico.VandeWeghe\}@UGent.be \\ Department of Geography \\ Ghent University \\ Krijgslaan 281 (S8) \\ B-9000 Ghent (Belgium)
}

\begin{abstract}
The widespread adoption of location-aware technologies has afforded analysts new opportunities for efficiently collecting trajectory data of moving individuals. These technologies enable measuring trajectories as a finite sample set of time-stamped locations. The uncertainty related to both finite sampling and measurement errors make it often difficult to reconstruct and represent a trajectory followed by an individual in space-time. Time geography offers an interesting framework to deal with the potential path of an individual in between two sample locations. While this potential path may be easily delineated for travels along networks, this will be less straightforward for more nonnetwork-constrained environments. Current models, however, have mostly concentrated on network environments on the one hand and do not account for the spatiotemporal uncertainties of input data on the other hand. This article simultaneously addresses both issues by developing a novel methodology to capture potential movement between uncertain space-time points in obstacle-constrained travel environments.
\end{abstract}

Keywords: time geography, space-time prism, uncertainty; obstacles; accessibility

\section{Introduction}

Recent years have seen the development of a range of widely and readily available tracking technologies, such as location-aware technologies (LATs) (2004) and geosensor networks (Stefanidis et al. 2003). These technologies are revolutionising the ways in which data about spatial behaviour is acquired by enabling researchers to collect massive volumes of trajectory data of mobile objects and individuals in real-time. Tracking data, however, are affected by at least two important sources of spatiotemporal uncertainty. First, trajectories are typically approximated by a sequence of locations pinpointed at discrete timestamps. Due to finite sampling, the uncertain positions of an individual have to be interpolated between successive 
sample points. While uncertainty about an individual's trajectory increases if sampling intervals are larger, higher sampling frequencies result in finer granularity and more spatiotemporal detail (Hornsby et al. 2002). The sampling frequency may be inherent to the tracking device at hand or may result from an incomplete spatial coverage of a geosensor network (i.e. the position of an individual is not recorded in areas outside the radio range of the sensors). In addition, sampling frequency can be influenced by system failures. For example, the sampling rate of GPS measurements may decrease in urban locales if the signal is blocked by obstructions (e.g. buildings). A second source of uncertainty arises from the fact that sample points themselves are prone to measurement inaccuracy depending on the spatial resolution of the tracking technique used. While individuals may be traced with an acceptable accuracy using GPS, the accuracy of short-range, wireless radio-communication technologies is often much lower and may depend upon the radio range and power class of the sensors and the amount of overlap between their radio ranges. Both finite sampling and measurement errors often hamper a straightforward reconstruction of individual trajectories on the basis of tracking data.

To cope with the problem of finite sampling in moving object databases (MODs), several researchers, among them (Sistla et al. 1998), (Moreira et al. 1999), (Trajcevski et al. 2004), and (Pfoser et al. 2005) have sought to delineate and query the unknown path between two observed locations given a presupposed maximum travel velocity in an unconstrained isotropic travel environment. In line with the advances in MODs, time geographers have also studied the sampling problem extensively using time geography's key concept, i.e. the space-time prism (Hägerstrand 1970, Miller 1991, Kwan et al. 1998, Yu et al. 2008). However, while the sampling problem is well-studied in time geography, the equally important problem of how this sampling problem interferes with the imperfect observation of sample points has received far less attention (Miller 2005). A notable exception is Neutens et al. (2007a) who, relying on the basic principles of rough set theory (Pawlak 1982), provide a conceptual framework to analyse how spatial and temporal uncertainty about the sample points propagates through a space-time prism by specifying lower and upper approximations of the prism dimensions. While conceptually appealing, their model has limited applicability since it assumes that travel occurs in an environment without any obstacles. The aim of the present paper is to enhance the applicability of this conceptual model to constrained travel environments and put it into practice by proposing and implementing a formal theoretical framework for defining and constructing rough space-time prisms in planar space with obstacles. The framework is particularly useful for modelling nonnetwork-constrained phenomena (e.g. pedestrian movement in urban and built environments) and accounts for both finite sampling and measurement errors.

The remainder of this article is organised as follows. Since our approach relies on time geography, the next section introduces the key concepts of time geography and documents the geocomputational models that have been developed in recent years for analysing an object's uncertain position between two fixed sample points. Section 3 discusses the formal definition and representation of a traditional space-time prism. This definition is extended toward the case of uncertain constraints in section 4, and the case of an environment populated with obstacles in section 5. Then in section 6, both approaches are combined, and an algorithm to derive obstacleconstrained space-time prisms with uncertain constraints is presented. An example case within a simple environment is used throughout the paper to clarify the methodology. Finally, in section 7 , we draw conclusions and outline avenues for future research. 


\section{Background}

Time geography

Back in the 1970s, Torsten Hägerstrand (1970) and his associates at the University of Lund (Sweden) developed a worldview for understanding the interdependencies between human beings, nature and technology, known as time geography. Time geography provides a conceptual perspective to analyse spatiotemporal patterns of human movement. In particular, the timegeographical approach articulates the scarcity of space and time, and emphasizes the importance of the constraints an individual is faced with when moving through geographical space (Pred 1977, Lenntorp 1978). Three types of constraints are distinguished: (i) Capability constraints refer to an individual's cognitive limitations and physiological necessities such as eating or sleeping; (ii) Coupling constraints restrict travel and activity participation by dictating where, when, and for how long individuals have to join other people, tools, or material artefacts in space and time; (iii) Authority constraints refer to the institutional and societal context including laws, rules, norms and other regulations implying that specific areas are only accessible at specific times for specific persons. These three constraints are interrelated and manifest themselves by dictating the time budget during which activities can be undertaken to achieve a project (i.e. a series of sequential tasks necessary to the completion of any intention-inspired or goal-oriented behaviour) (Carlstein et al. 1978, Pred 1981).

The basic tenet of time geography is the space-time path which represents the uninterrupted string of movements of an individual in space-time. The course of a space-time path results from the interaction between constraints and projects and is typically visualised in a three-dimensional framework in which time is integrated orthogonally to a flattened topography. In this representation, an individual's travel speed is inversely proportional to the slope of its space-time path. Another key concept is the space-time prism which demarcates the envelope of all spacetime paths an individual might have drawn during the time budget between two successive timestamps. It is important to note that while a space-time path represents revealed spatial behaviour, space-time prisms capture potential spatial behaviour.

\section{Implementations of time-geographical concepts}

In the past two decades, the time-geographical approach has regained attention in geographical information science and transportation geography. Technological advances in geographical information systems (GIS) as well as the increased availability of georeferenced trajectory data have opened up new opportunities to enhance the realism of the time-geographical entities and to apply these in empirical studies regarding individual accessibility (Miller 1991, Kwan et al. 1998, $\mathrm{Yu}$ et al. 2007, Schwanen et al. 2008). Renewed interest in time geography also dovetails with the paradigm shift in transportation policy towards travel demand management and the activitybased approach to travel forecasting that has increasingly gained momentum since the mid-70s (Axhausen et al. 1992, Timmermans et al. 2002, Dong et al. 2006).

\section{Modelling heterogeneous travel environments}

In recent years, there has been a flurry of geocomputational methods to model the unknown position of an individual during the time budget between two time-stamped positions. These methods have sought to improve the classical representation of the space-time prism to deal with the complexities of real-world travel environments. An important accomplishment is the 
calculation of potential path areas within transportation networks. Following the seminal work of Miller (1991), a number of authors have specified GIS-based algorithms to derive the paths that an individual could have taken between two discrete locations within a road network (e.g. Kwan et al. 1998, Miller et al. 2000, Wu et al. 2001, Weber et al. 2002, Kim et al. 2003). These network-based approaches offer only a static synopsis of an individual's travel possibilities but do not account for the spatial variation in travel possibilities during a time budget. Therefore, some authors have proposed algorithms to derive the full three-dimensional, network-based space-time prism based on shortest path algorithms within road networks (Neutens et al. 2007a, Kuijpers et al. 2009). Despite the proliferation of methods to delineate travel possibilities within transportation networks, only few studies have been concerned with modelling non-motorised, non-network yet spatially constrained movements through space-time prisms. A recent example is Miller and Bridwell (2009). They introduced an analytical theory to derive field-based spacetime paths and prisms using velocity fields. A velocity field is a smooth differential function that assigns a velocity to each location in continuous space (Puu et al. 1999). Although this method allows examining theoretical conjectures about accessibility in continuous space, a spatial decomposition into a lattice is required to use the approach in empirical research. A drawback of this decomposition is that it introduces errors that cannot be resolved by increasing the lattice density (see Goodchild 1977, Miller et al. 2009).

\section{Modelling travel constraint uncertainty}

Another line of scientific inquiry concerns the implications of spatiotemporal uncertainty about the prism properties (i.e. maximum travel velocity, origin and destination point) for the prism dimensions. For example, several researchers have examined the ways in which prism-based accessibility is affected by uncertainty in travel time caused by unreliable transportation or systematically recurring congestion (e.g. Hall 1983, Ettema et al. 2007, Schwanen et al. 2008). Hendricks et al. (Hendricks et al. 2003), for their part, have proposed a sequential partitioning method to model a wayfinder's indiscernibility between future travel possibilities. Neutens et al. (2007b) have furthered this approach and sought to calculate and represent the three-dimensional prism if its origin and destination points are not known exactly. They introduced the concept of a rough space-time prism to model the potential movement between two uncertain sample points through the prism's lower and upper approximation. Although a conceptually elegant solution to deal with both finite sampling and measurement errors, the application of the approach is currently limited to unconstrained travel environments. Furthermore, it does not explicitly address how measurement uncertainty about sample points intertwines with uncertainty about the maximum travel velocity.

The present paper contributes to these lines of inquiry in at least two ways. First, we complement existing network-based methods with a novel approach to model nonnetwork-constrained phenomena, including pedestrian movements in urban and built environments. Drawing on research in computational geometry (e.g. Kapoor et al. 1997, Hershberger et al. 1999, Inkulu et al. 2009), we propose a methodology to construct space-time prisms in planar space with obstacles. Our approach does not require a discretisation of space and time. Rather than approximating space-time prisms as a set of contours at discrete moments in time using a fieldbased lattice, space-time prisms are modelled and implemented as solid objects in continuous space. This eliminates errors resulting from discretisation and avoids the storage and processing of large amounts of voxel data. Second, the approach allows gaining insights into how 
combinations of uncertainty about the maximum travel velocities and the spatiotemporal uncertainty about sample points affect an individual's travel possibilities.

\section{A space-time prism in an unconstrained travel environment}

A space-time prism measures the ability to reach locations in space and time in between two locations separated in time, respectively denoted as origin and destination. Origins and destinations may be derived from the locations of fixed activities reported in travel diaries (e.g. Cullen et al. 1975, Weber et al. 2003), or they can be estimated using stochastic frontier modelling (e.g. Pendyala et al. 2002, Kitamura et al. 2006). As in (Miller 2005, Shoval et al. 2007), this article will take the viewpoint of origins and destinations sampled through a tracking system, although our method can be applied to spatiotemporal data obtained from other observation or estimation techniques as well. In classical time geography, a space-time prism is determined by its origin, destination, and a finite maximum velocity in an unconstrained isotropic travel environment (Miller 2005). Given these constraints, a space-time prism is obtained from the intersection of two cones (Figure 1). The forward cone encloses all space-time points that can be reached from the origin, while the backward cone captures all space-time points where an individual could have come from when (s)he is to arrive at the destination. In the remainder we will refer to these cones as reachability cones. The height of the reachability cones corresponds to the time budget that results from the origin and destination temporal coupling constraints. Their side slopes and aperture correspond to the maximum travel velocity that an individual may attain.

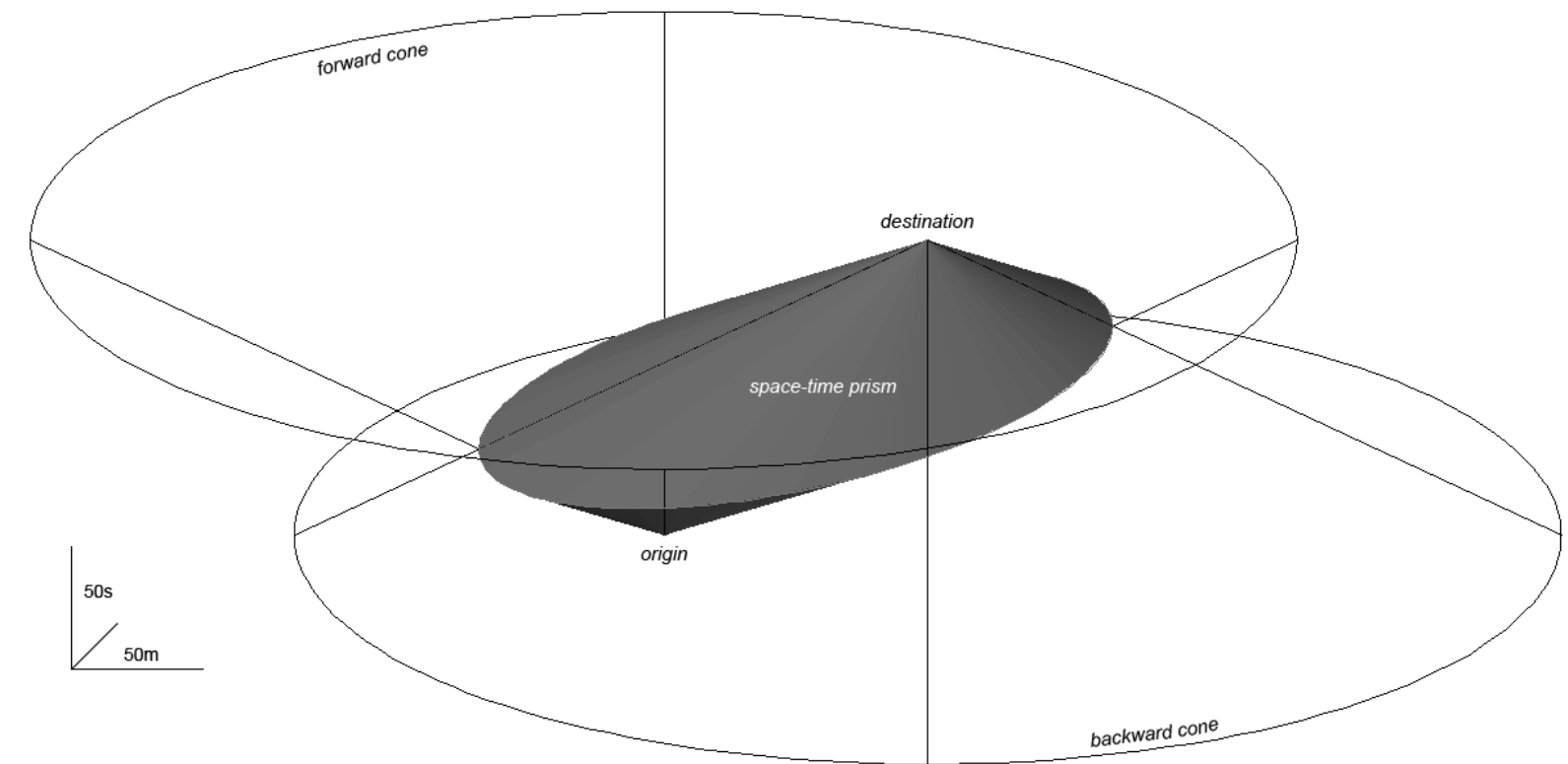

Fig. 1 Space-time prism obtained from the intersection of a forward cone and a backward cone

More formally, a space-time prism in an unconstrained isotropic travel environment can be defined as follows. Let $\mathbb{R}$ be the set of real numbers, $\mathbb{R}^{+}$the set of positive real numbers, and $\mathbb{R}^{2}$ the two-dimensional real plane with metric $d_{R}$ being the Euclidean distance. Though any metric space $S$ with metric $d_{s}$ would be possible, we will consider travel in the $(x, y)$-plane $\mathbb{R}^{2}$ and 
represent this movement in $(x, y, t)$-space $\mathbb{R}^{2} \times \mathbb{R}$, where $t$ represents time. Let $o=\left(x_{o}, y_{o}, t_{o}\right) \in$ $\mathbb{R}^{2} \times \mathbb{R}$ denote the origin, $d=\left(x_{d}, y_{d}, t_{d}\right) \in \mathbb{R}^{2} \times \mathbb{R}$ the destination, $t b \in \mathbb{R}^{+}$the time budget, and $v_{\max } \in \mathbb{R}^{+}$the maximum velocity.

Definition 1. The forward cone $F C\left(o, t b, v_{\max }\right)$ with origin $o$, time budget $t b$, and maximum velocity $v_{\max }$ is the set of all space-time points $p=(x, y, t)$ that satisfy:

$$
\left\{\begin{array}{c}
d_{R}(o, p) \leq\left(t-t_{o}\right) v_{\max } \\
t_{o} \leq t \leq t_{o}+t b
\end{array}\right.
$$

The forward cone has its apex at the origin and is oriented forward in time.

Definition 2. The backward cone $B C\left(d, t b, v_{\max }\right)$ with destination $d$, time budget $t b$, and maximum velocity $v_{\max }$ is the set of all space-time points $p=(x, y, t)$ that satisfy:

$$
\left\{\begin{array}{c}
d_{R}(d, p) \leq\left(t_{d}-t\right) v_{\max } \\
t_{d}-t b \leq t \leq t_{d}
\end{array}\right.
$$

The backward cone has its apex at the destination and is oriented backward in time.

Definition 3. The space-time prism $P\left(o, d, v_{\max }\right)$ with origin $o$, destination $d$, and maximum velocity $v_{\max }$ is given by:

$$
P\left(o, d, v_{\max }\right)=F C\left(o, t_{d}-t_{o}, v_{\max }\right) \cap B C\left(d, t_{d}-t_{o}, v_{\max }\right)
$$

Fig. 1 shows how $P\left(o, d, 2 \mathrm{~ms}^{-1}\right)$ for $o=(0 \mathrm{~m}, 0 \mathrm{~m}, 0 \mathrm{~s}), d=(100 \mathrm{~m}, 0 \mathrm{~m}, 120 \mathrm{~s})$ is obtained from the intersection of reachability cones. In the remainder, we will extend the space-time prism to cope with uncertain origins, destinations, and maximum velocities, and with obstacle-constrained travel environments.

\section{A rough space-time prism in an unconstrained environment}

In order to model the uncertainty of constraints, each space-time prism $P$ will be represented as a rough set through its lower and upper (approximation) prism. The upper prism $P_{U}$ includes all space-time locations that are potentially reachable. $P_{U}$ is delimited by the least restricted spacetime paths in terms of accessibility, i.e. best case scenario paths. Suppose that there is uncertainty about the departure time (temporal coupling constraint) of an individual. Then $P_{U}$ will be bounded by space-time paths that assume the earliest possible departure time. Analogously, the lower prism represents what is reachable in all cases and is bounded by worst case scenario space-time paths (e.g. assuming the latest possible departure time). The uncertain part of a rough space-time prism is the boundary body $\Delta P_{L}$, which equals $P_{U} \backslash P_{L}$. Hence, three parts can be distinguished: what is certainly reachable $\left(P_{L}\right)$, what may be reachable $\left(\Delta P_{L}\right)$, and what is certainly not reachable $\left(\backslash P_{U}\right)$. Though this distinction has to be kept in mind, we will not explicitly consider $\Delta P_{L}$ any further, due to its dependency on $P_{L}$ and $P_{U}$. In the remainder of this paper, we will use the term rough to refer to the dual representation of a lower and upper approximation.

Rough space-time prisms can deal with three types of uncertainty, i.e. spatial, temporal and velocity uncertainty (Neutens et al. 2007a). In the context of tracking systems, there is spatial and temporal uncertainty stemming from the measurement inaccuracy of the tracking technologie. 
Wireless tracking technologies such as Bluetooth and WiFi employ a certain spatial radio range and temporal scanning interval. Although uncertainty may differ in space and time, for many tracking data it makes sense to presume a constant spatial and temporal uncertainty related to the accuracy of the technology at hand. The maximum velocity, on the other hand, cannot be directly related to measurement accuracy and is often approximated by means of a lower and an upper estimate (e.g. maximum velocity on a road during respectively peak and off-peak hours).

Consider origin $o$, destination $d$, time budget $t b$, spatial accuracy $\delta s$, temporal accuracy $\delta t$, maximum velocity $v_{\max }$, lower maximum velocity $v_{L}$, and upper maximum velocity $v_{U}$, with $\delta s, \delta t, v_{L}, v_{U} \in \mathbb{R}^{+}$, and $v_{L} \leq v_{\max } \leq v_{U}$.

Definition 4. The lower forward cone $F C_{L}\left(o, t b, \delta s, \delta t, v_{L}\right)$ is the set of all space-time points $p=(x, y, t)$ that satisfy:

$$
\left\{\begin{array}{c}
d_{R}(o, p) \leq\left(t-t_{o}-\delta t\right) v_{L}-\delta s \\
t_{o}+\delta t \leq t \leq t_{o}+t b-\delta t
\end{array}\right.
$$

Definition 5. The upper forward cone $F C_{U}\left(o, t b, \delta s, \delta t, v_{U}\right)$ is the set of all space-time points $p=(x, y, t)$ that satisfy:

$$
\left\{\begin{array}{c}
d_{R}(o, p) \leq\left(t-t_{o}+\delta t\right) v_{U}+\delta s \\
t_{o}-\delta t \leq t \leq t_{o}+t b+\delta t
\end{array}\right.
$$

Definition 6. The lower backward cone $B C_{L}\left(d, t b, \delta s, \delta t, v_{L}\right)$ is the set of all space-time points $p=(x, y, t)$ that satisfy:

$$
\left\{\begin{array}{c}
d_{R}(d, p) \leq\left(t_{d}-\delta t-t\right) v_{L}-\delta s \\
t_{d}-t b+\delta t \leq t \leq t_{d}-\delta t
\end{array}\right.
$$

Definition 7. The upper backward cone $B C_{U}\left(d, t b, \delta s, \delta t, v_{U}\right)$ is the set of all space-time points $p=(x, y, t)$ that satisfy:

$$
\left\{\begin{array}{c}
d_{R}(d, p) \leq\left(t_{d}+\delta t-t\right) v_{U}+\delta s \\
t_{d}-t b-\delta t \leq t \leq t_{d}+\delta t
\end{array}\right.
$$

Definition 8. The lower space-time prism $P_{L}\left(o, d, \delta s, \delta t, v_{L}\right)$ is given by:

$$
P_{L}\left(o, d, \delta s, \delta t, v_{L}\right)=F C_{L}\left(o, t b, \delta s, \delta t, v_{L}\right) \cap B C_{L}\left(d, t b, \delta s, \delta t, v_{L}\right)
$$

Definition 9. The upper space-time prism $P_{U}\left(o, d, \delta s, \delta t, v_{U}\right)$ is given by:

$$
P_{U}\left(o, d, \delta s, \delta t, v_{U}\right)=F C_{U}\left(o, t_{d}-t_{o}, \delta s, \delta t, v_{U}\right) \cap B C_{U}\left(d, t_{d}-t_{o}, \delta s, \delta t, v_{U}\right)
$$

Property 1. $\forall P\left(o, d, v_{\max }\right) \subset \mathbb{R}^{2} \times \mathbb{R}, \forall v_{L}, v_{U}, \delta s, \delta t \in \mathbb{R}^{+}$

$\exists P_{L}\left(o, d, \delta s, \delta t, v_{L}\right), P_{U}\left(o, d, \delta s, \delta t, v_{U}\right) \subset \mathbb{R}^{2} \times \mathbb{R}: P \supseteq P_{L} \wedge P \subseteq P_{U} \wedge v_{L} \leq v_{\max } \leq v_{U}$

That is, for each space-time prism $P$ and for each set of valid rough maximum velocities, spatial accuracy, and temporal accuracy, there exist a lower space-time prism $P_{L}$ and an upper spacetime prism $P_{U}$, such that $P$ contains $P_{L}$, and $P_{U}$ contains $P$. Note that $P_{L}$ might be the empty set independent of the uncertainty parameters, whereas $P_{U}$ can never be an empty set whenever one of these parameters is strictly positive. The model of rough space-time prisms also generalises the classical model, which is obtained from the special case where accuracies are negligible and 
rough maximum velocities are considered equal. Therefore, the boundary body dissolves and, according to definition 9, the attained upper and lower prisms both equal the classic prism. In addition, note that, according to the first equation of definition 5, the upper forward cone has its apex at time $t=t_{o}-\delta t-\delta s / v_{U}$. However, due to the second equation, only time stamps higher than or equal to $t_{o}-\delta t$ are valid. Analogous reasoning applies for the upper backward cone, and therefore, upper reachability cones are flattened at the top over a circular area with radius $\delta s$ which reflects the underlying spatial uncertainty.

The example approximation prisms $P_{L}\left(o, d, 10 \mathrm{~m}, 5 \mathrm{~s}, 1.9 \mathrm{~ms}^{-1}\right)$ and $P_{U}\left(o, d, 10 \mathrm{~m}, 5 \mathrm{~s}, 2.1 \mathrm{~ms}^{-1}\right)$ are illustrated in Fig. 3 (with $o, d$ as in Fig. 1).

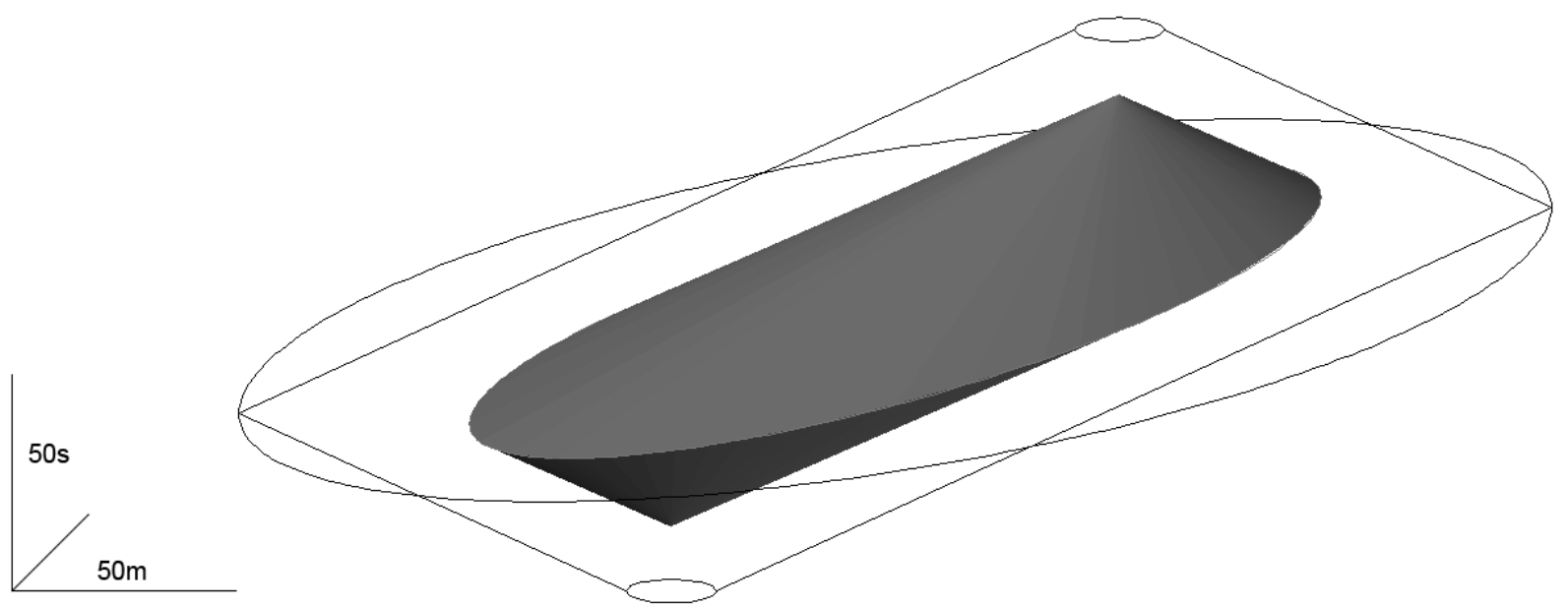

Fig. 2 An uncertain space-time prism modelled by its lower (in grey), and upper (filled lines) approximation.

\section{A space-time prism in an obstacle-constrained travel environment}

Until now, movement has been considered to happen in an unconstrained travel environment. Though this assumption underlies traditional time geography, it is hardly tenable and most often highly unrealistic for true geographical spaces. This assumption has been abandoned in later work, as discussed in section 2. In addition to these approaches, we present an alternative considering an isotropic travel space populated with obstacles. Obstacles can be any kind of inaccessible areas, as are building blocks, water bodies and highways to pedestrians. The space in between the obstacles is assumed to be unconstrained and isotropic, which enables us to preserve the maximum velocity constraint and thereby support the well-studied time-geographical entities introduced earlier.

We will clarify our approach using a simple example case. Fig. 3 shows a map of three buildings $A, B$, and $C$ at university campus 'De Sterre' in Ghent (Belgium). The area surrounding the buildings can be assumed open and accessible to pedestrians. Two positions are located at building entrances, for which we assume they are a student's origin $o$ and destination $d$ in between two subsequent lectures. Let us consider a time budget of two minutes for the student to walk from $o$ to $d$, with a maximum walking velocity of $2 \mathrm{~m} . \mathrm{s}^{-1}$ as an educated guess. Our aim is now to construct the student's space-time prism according to these constraints, taking account of the obstacles blocking his/her passage. 


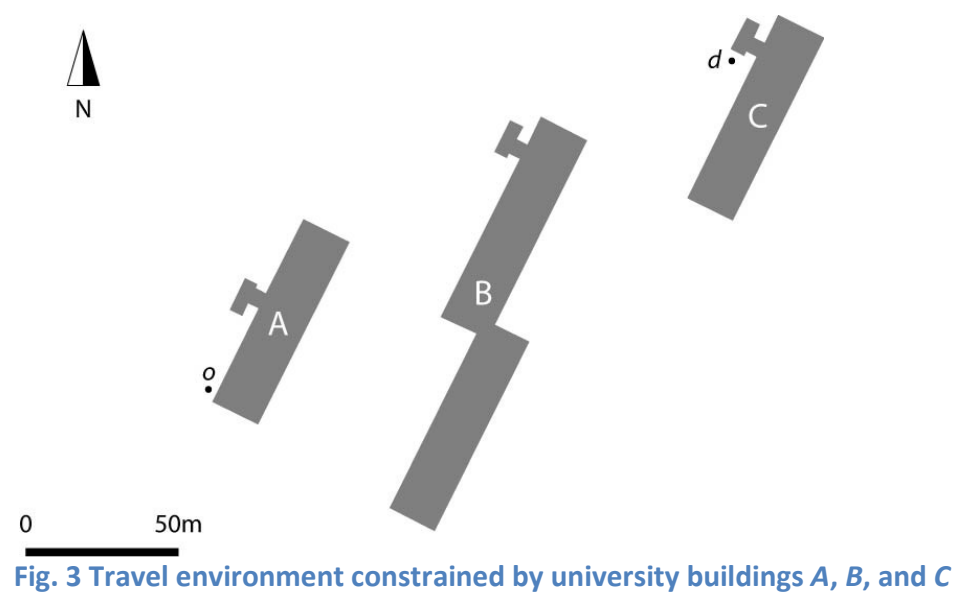

As follows from section 3, reachability cones provide an answer to two fundamental questions: (i) which locations are reachable for the individual within the given time budget if (s)he starts at the origin; (ii) from which locations is the destination reachable within the given time budget (2). Assessing the accessibility from (to) a certain location requires knowledge about all shortest paths from (to) this place. In an unconstrained isotropic space, all reachable locations lie within a certain radius from the origin or destination, as all shortest paths are simply the straight beeline connectors. To construct space-time prisms in obstacle-constrained environments, however, shortest paths are to be calculated that avoid the obstacles.

In computational geometry and geographical information systems, obstacles such as buildings and impassable areas are generally modelled as regions using a polygonal geometry. Research in computational geometry has offered efficient algorithms to compute the shortest paths in a Euclidean plane in the presence of such polygonal obstacles. To this end, there have been two fundamentally different approaches. The visibility graph method (Kapoor et al. 1988, Kapoor et al. 1997), on the one hand, and the wavefront method (Mitchell 1993, Hershberger et al. 1999) on the other hand. Some (e.g. Inkulu et al. 2009) have also considered combinations thereof. For exact algorithms and computational details, we refer to the specialised literature. We may employ such an algorithm in order to determine all necessary shortest paths within an obstacleconstrained travel environment in case of obstacles modelled as polygons, as we will further assume according to its generality in GIS. It is important to note that only the shortest paths to polygon vertices have to be calculated, due to the following reasoning. Whenever an obstacle $q$ blocks the straight connection from $o$ to any other point, the corresponding shortest path(s) from $o$ will pass along an extreme (i.e. a tangential point) of $q$ when observed from $o$. This extreme will always be a vertex in the case of a polygonal obstacle.

Let $Q=\left\{q_{1}, \ldots, q_{i}, \ldots, q_{N}\right\}$ be a set of obstacles, and $V_{i}=\left\{v_{i 1}, \ldots, v_{i j}, \ldots, v_{i M}\right\}$ be the set of vertices of obstacle $q_{i}$. Let $S P(o, v, Q)$ denote the shortest path from $o$ to $v$ avoiding the obstacles in $Q$.

Definition 10. The shortest path set $\operatorname{SPS}(o, Q)$ is the set of all shortest paths $\operatorname{SP}(o, v, Q)$ from $o$ to all vertices $v$ of all obstacles $q$ in $Q$ : 


$$
\operatorname{SPS}(o, Q)=\bigcup_{i=1}^{N} \bigcup_{j=1}^{M} S P\left(o, v_{i j}, Q\right)
$$

Fig. 4 and Fig. 5 respectively show a map of $\operatorname{SPS}(o,\{A, B, C\})$ and $S P S(d,\{A, B, C\})$ for the example case. An SPS is a tree in which each vertex $v$ is a node that is associated with a shortest path distance and a parent (i.e. the preceding vertex along the shortest path), with the origin / destination being the root parent. A vertex is reachable if its shortest path distance is smaller than or equal to the distance budget, i.e. the product of time budget and maximum velocity.

Let $d_{X}(v)$ denote the distance to vertex $v$ along shortest path $X$.

Definition 11. The reachable set $R S\left(o, Q, t b, v_{\max }\right)$ is the set of all vertices that lie within distance budget $t b \cdot v_{\max }$ from origin $o$ along a shortest path avoiding the obstacles in $Q$ :

$$
R S\left(o, Q, t b, v_{\max }\right)=\left\{v \mid d_{S P(o, v, Q)}(v) \leq t b \cdot v_{\max }\right\}
$$

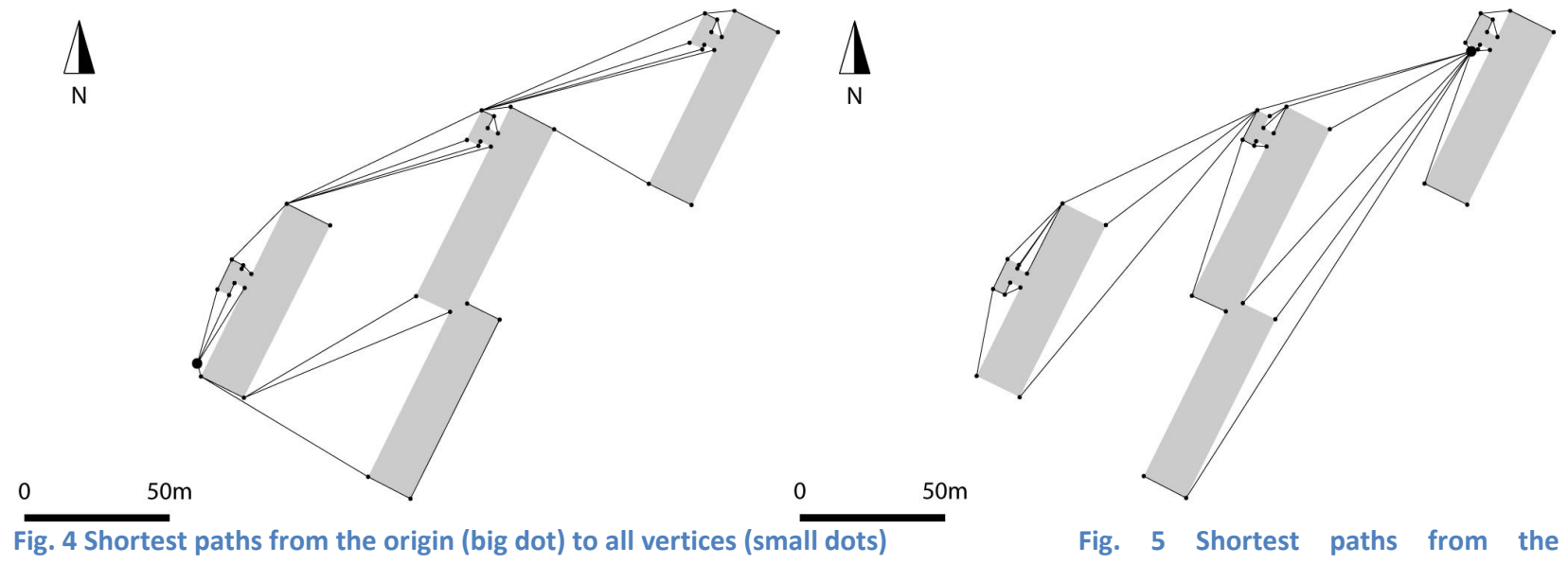
destination (big dot) to all vertices (small dots)

All parent vertices act as wavefront propagators that induce separate reachability cones according to the time budget that remains at the time they are reached. Given a set of obstacles $Q$, a time budget $t b$, and a maximum velocity $v_{\max }$, the forward cone at parent vertex $v=(x, y, t)$ is specified as $F C\left(v, t b-t, v_{\max }\right)$ with $t=d_{S P(o, v, Q)}(v) \cdot v_{\max }{ }^{-1}$. Analogous reasoning applies for a parent's backward cone $B C\left(v, t b-t, v_{\max }\right)$ and $t=d_{S P(d, v, Q)}(v) \cdot v_{\max }{ }^{-1}$.

Not all parts of the yet obtained parent cones are reachable. Their inaccessible parts can be modelled as spatial zones that are to be extruded vertically along the time axis. Two different types of extrusions have to be made: (i) extrusion of the areas that overlap with obstacles; (ii) extrusion of the areas that are obscured by obstacles. To obtain their reachable parts, the extrusions will have to be subtracted from the parent cones. As thereafter, the resulting bodies are no longer true cones, we will term them reachability bodies, i.e. forward body and backward body. 
Let $L(a, b)$ denote straight spatial connection line segment from $a$ to $b$.

Definition 12. The parent forward body $\operatorname{PFB}\left(v, o, Q, t b, v_{\max }\right)$ for a parent $v$ with respect to origin $o$, obstacle set $Q$, time budget $t b$, and maximum velocity $v_{\max }$ is given by:

$$
\begin{aligned}
\operatorname{PFB}(v, o, Q, t b & \left., v_{\max }\right) \\
& =F C\left(v, t b-d_{S P(o, v, Q)}(v) \cdot v_{\max }{ }^{-1}, v_{\max }\right) \\
& \backslash\{p=(x, y, t) \mid \forall q \in Q: L(v, p) \cap q=\emptyset\}
\end{aligned}
$$

Definition 13. The parent backward body $P B B\left(v, d, Q, t b, v_{\max }\right)$ for a parent $v$ with respect to destination $d$, obstacle set $Q$, time budget $t b$, and maximum velocity $v_{\text {max }}$ is given by:

$$
\begin{aligned}
P B B\left(v, d, Q, t b, v_{\max }\right) & \\
& =B C\left(d, t b-d_{S P(d, v, Q)}(v) \cdot v_{\max }{ }^{-1}, v_{\max }\right) \\
& \backslash\{p=(x, y, t) \mid \forall q \in Q: L(v, p) \cap q=\emptyset\}
\end{aligned}
$$

Fig. 6 and

Fig. 7 illustrate the reachability bodies for a parent vertex of building $B$, according to origin, destination and time budget specified earlier. The figures also indicate the footprint of the obstructed zones to be extruded. Note that the reachability bodies are situated at different time intervals, due to their different temporal orientation as well as to the temporal difference corresponding to the respective shortest path distances from $o$ to $v$ and from $d$ to $v$.

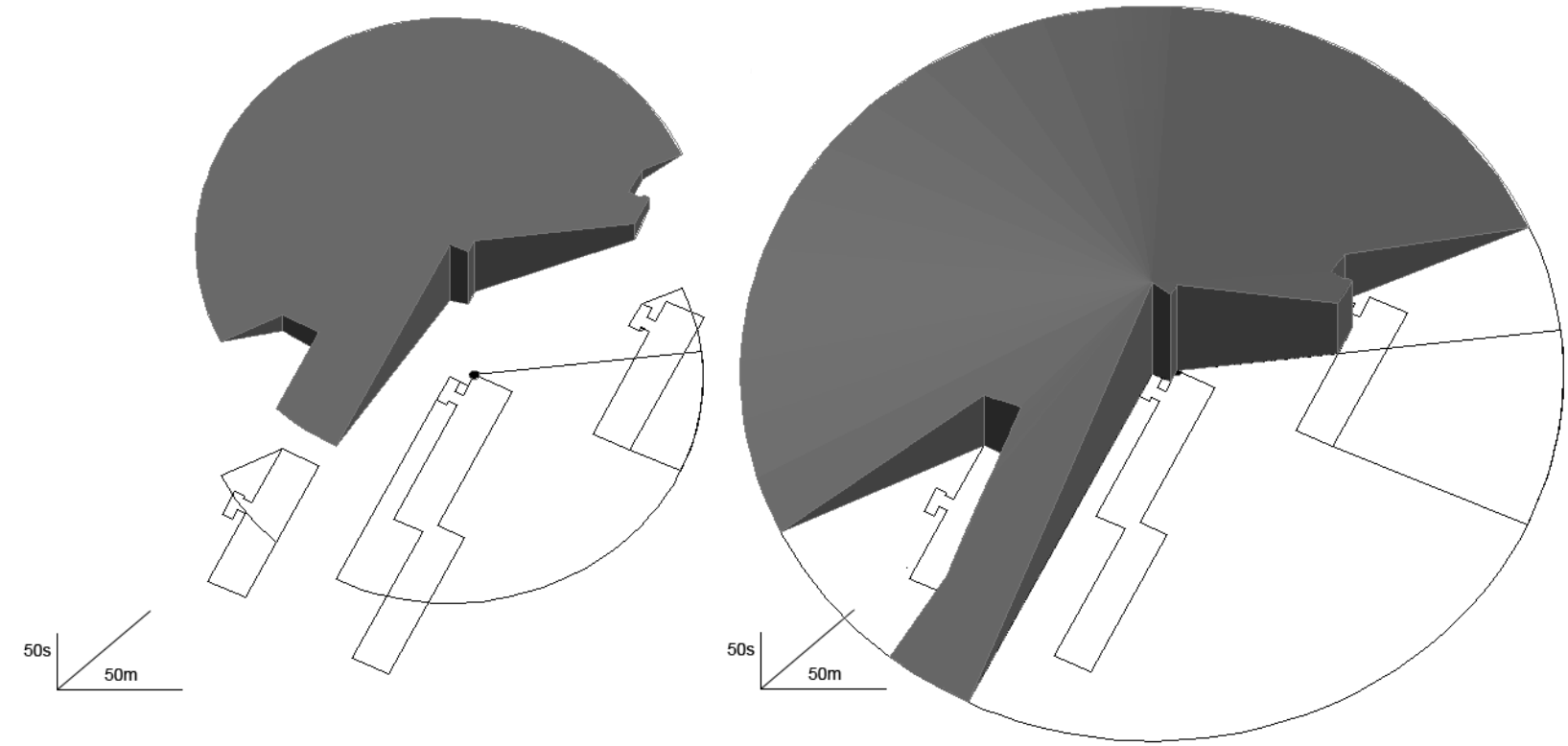

Fig. 6 Forward reachability body of a parent vertex (black dot) with indication of spatial extrusion zones.

Fig. 7 Backward reachability body of a parent vertex (black dot) with indication of spatial extrusion zones.

The overall reachability bodies are now obtained from the union of all reachability bodies, either the forward or the backward bodies, over all parents.

Let $\operatorname{par}_{X}(v)$ denote the parent of vertex $v$ along shortest path $X$. 
Definition 14. The forward body $F B\left(o, Q, t b, v_{\max }\right)$ with origin $o$, obstacle set $Q$, time budget $t b$, and maximum velocity $v_{\max }$ is given by:

$$
\begin{array}{r}
F B\left(o, Q, t b, v_{\max }\right) \\
=\bigcup\left\{P F B\left(v_{p}, o, Q, t b, v_{\text {max }}\right) \mid \exists v \in R S\left(o, Q, t b, v_{\text {max }}\right): v_{p}=\operatorname{par}_{S P(o, v, Q)}(v)\right\}
\end{array}
$$

Definition 15. The backward body $B B\left(d, Q, t b, v_{\max }\right)$ with destination $d$, obstacle set $Q$, time budget $t b$, and maximum velocity $v_{\text {max }}$ is given by:

$B B\left(d, Q, t b, v_{\max }\right)$

$$
=\bigcup\left\{P B B\left(v_{p}, d, Q, t b, v_{\max }\right) \mid \exists v \in R S\left(d, Q, t b, v_{\max }\right): v_{p}=\operatorname{par}_{S P(d, v, Q)}(v)\right\}
$$

In analogy to definition 3, the obstacle-constrained space-time prism is obtained from the intersection of the forward and backward bodies (Fig. 8).

Definition 16. The obstacle-constrained space-time prism $\operatorname{OCP}\left(o, d, Q, v_{\max }\right)$ with origin $o$, destination $d$, obstacle set $Q$, and maximum velocity $v_{\text {max }}$ is given by:

$$
O C P\left(o, d, Q, v_{\max }\right)=F B\left(o, Q, t_{d}-t_{o}, v_{\max }\right) \cap B B\left(d, Q, t_{d}-t_{o}, v_{\max }\right)
$$

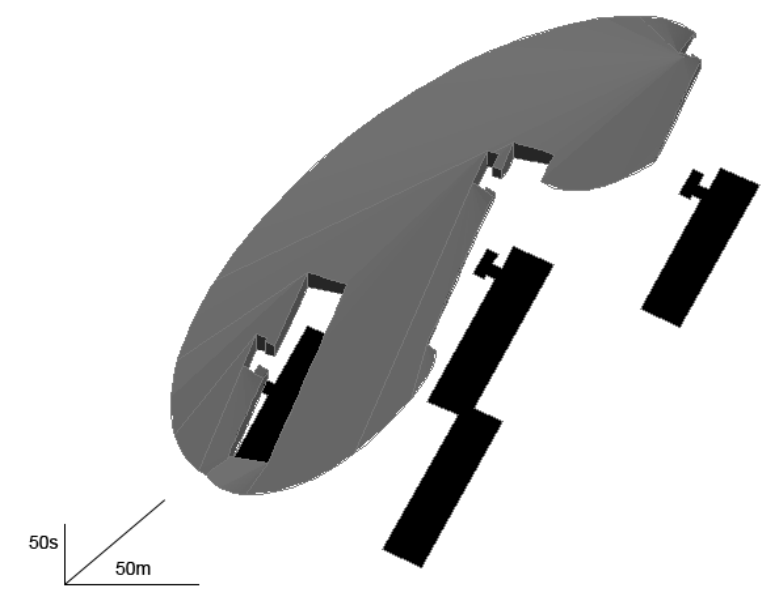

Fig. 8 Obstacle-constrained space-time prism with indication of obstacles

\section{A rough space-time prism in an obstacle-constrained travel environment}

\subsection{Combination of approaches}

This section concerns the integration of the approaches of sections 4 and 5. Whereas in a classical unconstrained environment, space-time prisms follow from the intersection of two reachability cones (definitions 1-3), two sets of parent reachability bodies are to be intersected, when accounting for obstacles (definitions 14-16). These reachability bodies are geometrically equivalent to cones with subtracted vertical extrusions (section 5). The constraints that determine these underlying cones, however, are not affected by the further subtraction of parts, and 
subsequent union with other bodies. Therefore, we may preserve the methodology of section 5 and adopt definitions 12 and 13, in order to obtain rough parent reachability bodies. Subsequently, the definitions 14-16 can be adapted analogously in order to construct the rough reachability bodies and space-time prisms for an environment constrained by obstacles. Hence, for a given origin $o$, destination $d$, obstacle set $Q$, time budget $t b$, spatial accuracy $\delta s$, temporal accuracy $\delta t$, lower maximum velocity $v_{L}$, and upper maximum velocity $v_{U}$, we obtain:

Definition 17. The lower parent forward body $P F B_{L}\left(v, o, Q, t b, \delta s, \delta t, v_{L}\right)$ for a parent $v$ is given by:

$$
\begin{aligned}
P F B_{L}(v, o, Q, & \left.t b, \delta s, \delta t, v_{L}\right) \\
& =F C_{L}\left(v, t b-d_{S P(o, v, Q)}(v) \cdot v_{L}^{-1}, \delta s, \delta t, v_{L}\right) \\
& \backslash\{p=(x, y, t) \mid \forall q \in Q: L(v, p) \cap q=\emptyset\}
\end{aligned}
$$

Definition 18. The upper parent forward body $\operatorname{PFB}_{U}\left(v, o, Q, t b, \delta s, \delta t, v_{U}\right)$ for a parent $v$ given by:

$$
\begin{aligned}
\operatorname{PFB}_{U}\left(v, o, Q, t b, \delta s, \delta t, v_{U}\right) & \\
& =F C_{U}\left(v, t b-d_{S P(o, v, Q)}(v) \cdot v_{U}^{-1}, \delta s, \delta t, v_{U}\right) \\
& \backslash\{p=(x, y, t) \mid \forall q \in Q: L(v, p) \cap q=\emptyset\}
\end{aligned}
$$

Definition 19. The lower parent backward body $P B B_{L}\left(v, d, Q, t b, \delta s, \delta t, v_{L}\right)$ for a parent $v$ is given by:

$$
\begin{aligned}
P B B_{L}(v, d, Q, & \left.t b, \delta s, \delta t, v_{L}\right) \\
& =B C_{L}\left(v, t b-d_{S P(d, v, Q)}(v) \cdot v_{L}^{-1}, \delta s, \delta t, v_{L}\right) \\
& \backslash\{p=(x, y, t) \mid \forall q \in Q: L(v, p) \cap q=\varnothing\}
\end{aligned}
$$

Definition 20. The upper parent backward body $P B B_{U}\left(v, d, Q, t b, \delta s, \delta t, v_{U}\right)$ for a parent $v$ is given by:

$$
\begin{aligned}
P B B_{U}(v, d, Q, & \left.t b, \delta s, \delta t, v_{U}\right) \\
& =B C_{U}\left(v, t b-d_{S P(d, v, Q)}(v) \cdot v_{U}{ }^{-1}, \delta s, \delta t, v_{U}\right) \\
& \backslash\{p=(x, y, t) \mid \forall q \in Q: L(v, p) \cap q=\emptyset\}
\end{aligned}
$$

Definition 21. The lower forward body $F B_{L}\left(o, Q, t b, \delta s, \delta t, v_{L}\right)$ is given by:

$F B_{L}\left(o, Q, t b, \delta s, \delta t, v_{L}\right)$

$$
=\bigcup\left\{P F B_{L}\left(v, o, Q, t b, \delta s, \delta t, v_{L}\right) \mid \exists v \in R S(o, Q, t b): v_{p}=\operatorname{par}_{S P(o, v, Q)}(v)\right\}
$$

Definition 22. The upper forward body $F B_{U}\left(o, Q, t b, \delta s, \delta t, v_{U}\right)$ is given by: $F B_{U}\left(o, Q, t b, \delta s, \delta t, v_{U}\right)$

$$
=\bigcup\left\{P F B_{U}\left(v, o, Q, t b, \delta s, \delta t, v_{U}\right) \mid \exists v \in R S\left(o, Q, t b, v_{U}\right): v_{p}=\operatorname{par}_{S P(o, v, Q)}(v)\right\}
$$

Definition 23. The lower backward body $B B_{L}\left(d, Q, t b, \delta s, \delta t, v_{L}\right)$ is given by: $B B_{L}\left(d, Q, t b, \delta s, \delta t, v_{L}\right)$

$$
=\bigcup\left\{P B B_{L}\left(v_{p}, d, Q, t b, \delta s, \delta t, v_{L}\right) \mid \exists v \in R S\left(d, Q, t b, v_{L}\right): v_{p}=\operatorname{par}_{S P(d, v, Q)}(v)\right\}
$$


Definition 24. The upper backward body $B B_{U}\left(d, Q, t b, \delta s, \delta t, v_{U}\right)$ is given by: $B B_{U}\left(d, Q, t b, \delta s, \delta t, v_{U}\right)$

$$
=\bigcup\left\{P B B_{U}\left(v_{p}, d, Q, t b, \delta s, \delta t, v_{U}\right) \mid \exists v \in R S\left(d, Q, t b, v_{U}\right): v_{p}=\operatorname{par}_{S P(d, v, Q)}(v)\right\}
$$

Definition 25. The lower obstacle-constrained space-time prism $\operatorname{OCP}_{L}\left(o, d, Q, \delta s, \delta t, v_{L}\right)$ is given by:

$$
O C P_{L}\left(o, d, Q, \delta s, \delta t, v_{L}\right)=F B_{L}\left(o, Q, t b, \delta s, \delta t, v_{L}\right) \cap B B_{L}\left(d, Q, t b, \delta s, \delta t, v_{L}\right)
$$

Definition 26. The upper obstacle-constrained space-time prism $\operatorname{OCP}_{U}\left(o, d, Q, \delta s, \delta t, v_{U}\right)$ is given by:

$$
O C P_{U}\left(o, d, Q, \delta s, \delta t, v_{U}\right)=F B_{U}\left(o, Q, t b, \delta s, \delta t, v_{U}\right) \cap B B_{U}\left(d, Q, t b, \delta s, \delta t, v_{U}\right)
$$

\subsection{Algorithm}

Based on the methodology of section 5 and the definitions 17-26, we have implemented an application program that takes an origin, a destination, a set of obstacles, a spatial accuracy, a temporal accuracy, a lower maximum velocity, and an upper maximum velocity as input parameters, and returns the corresponding rough obstacle-constrained space-time prisms. The resulting prisms are then visualised as 3D solids by means of a CAD system. A description of the application's main algorithm is given in pseudo-code in Algorithm 1. The algorithm first computes the shortest paths from the origin (oSPS) and from the destination ( $d S P S$ ), relying on an existing algorithm as discussed in section 5. Next, these shortest path sets are used to compute the reachable sets of obstacle vertices according to the time budget and maximum velocity for the lower (oLRS, $d L R S)$ and upper (oURS, $d U R S$ ) approximations. Then, the reachability bodies $\left(F B_{L}, F B_{U}, B B_{L}, B B_{U}\right)$ corresponding to the four reachable sets are calculated. According to definitions 21-24, this is achieved as the union of the respective parent bodies over all parents in the reachable set. As follows from definitions 17-20, these parent bodies are obtained as cones subtracted with extrusions of obstructed areas. Finally, forward and backward bodies are intersected to achieve the overall lower $\left(O C P_{L}\right)$ and upper space-time prisms $\left(O C P_{U}\right)$.

Algorithm 1 - Main algorithm for computation of rough obstacle-constrained space-time prisms

Inputs:

$$
o, d, Q, \delta s, \delta t, v_{L}, v_{U}
$$

Outputs:

$$
\begin{aligned}
& O C P_{L}\left(o, d, Q, \delta s, \delta t, v_{L}\right) \\
& O C P_{U}\left(o, d, Q, \delta s, \delta t, v_{U}\right)
\end{aligned}
$$

Algorithm:

01: $\quad$ shortest paths from $o \rightarrow o S P S=\operatorname{SPS}(o, Q)$

02: $\quad$ shortest paths from $d \rightarrow d S P S=\operatorname{SPS}(d, Q)$

03: $\quad t b=t_{d}-t_{o}$

04: $\quad$ using $o S P S \rightarrow o L R S=R S\left(o, Q, t b, v_{L}\right)$

05: $\quad$ using $d S P S \rightarrow d L R S=R S\left(d, Q, t b, v_{L}\right)$

06: $\quad$ using $o S P S \rightarrow o U R S=R S\left(o, Q, t b, v_{U}\right)$ 


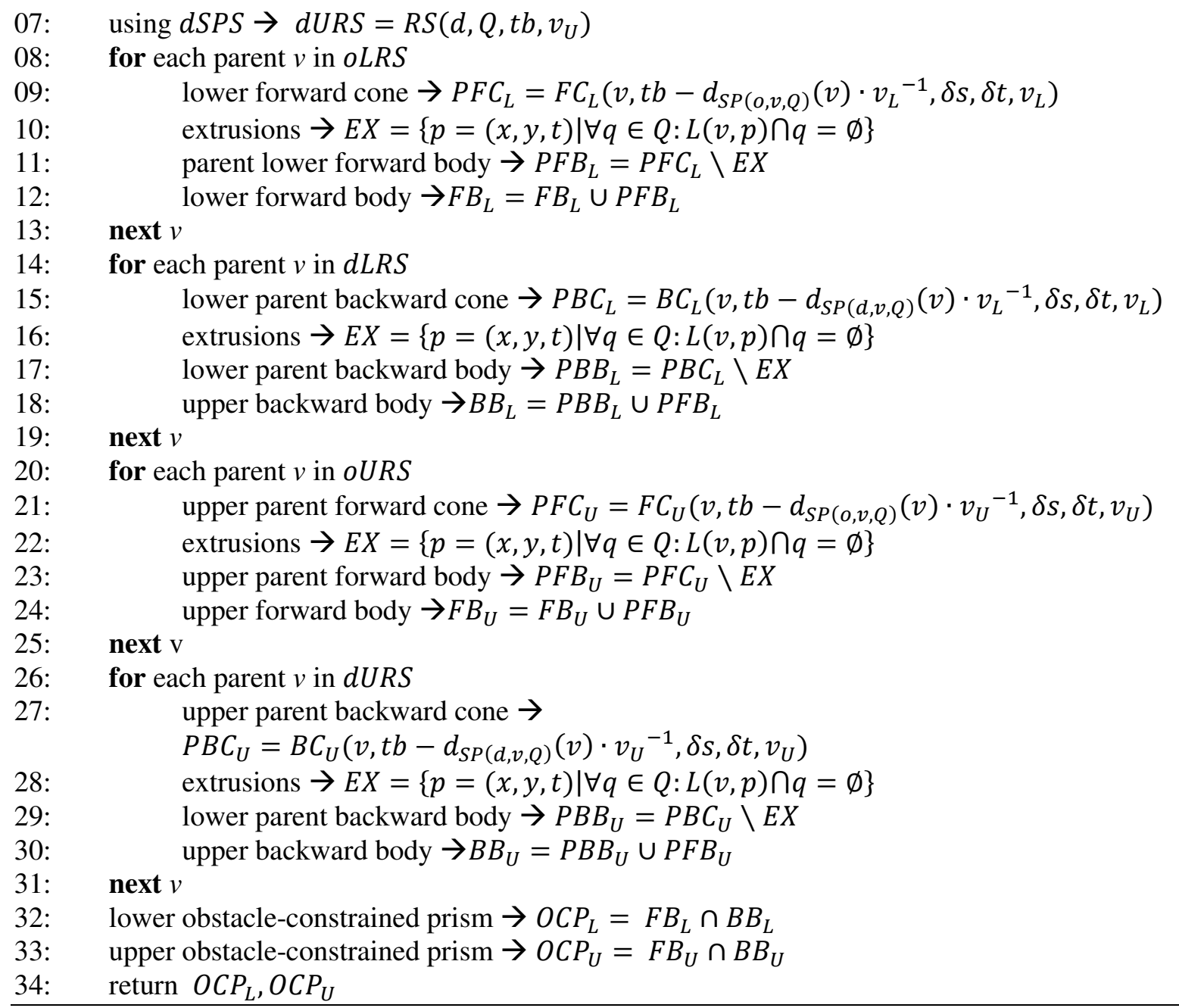

\subsection{Example}

To illustrate our methodology, we will reconsider the university campus example with a student having two minutes to travel from $o$ to $d$ (Fig. 3). Suppose that (s)he was tracked at $o$ and $d$ with a spatial accuracy $\delta s$ of $10 \mathrm{~m}$ and a temporal accuracy $\delta t$ of $5 \mathrm{~s}$. According to Bohannon (1997), reliable estimates for an adult's maximum gait speed range from $1.749 \mathrm{~m} . \mathrm{s}^{-1}$ to $2.533 \mathrm{~m} . \mathrm{s}^{-1}$ when considering differences in sex and age class. Let us take this as lower $v_{L}$ and upper $v_{U}$ approximation maximum velocity respectively. The lower and upper prisms corresponding to these constraints are presented in Fig. 9 and Fig. 10. A cross section through both prisms along the origin-destination axis is shown in Fig. 12. Note that, according to the definitions and properties of section 4, the temporal extremes of the prism lie strictly within the time budget for the lower approximation, whereas they exceed the time budget in the upper approximation. Also, the upper prism is flattened out at its origin and destination, due to the spatial uncertainty.

It appears that there is a large difference between the lower and upper prisms in this case: whereas the student might have easily passed along all sides of all buildings in the upper prism, (s)he is restricted to an almost linear course passing north of the buildings in the lower approximation scenario. Hence, it would have been a harmful limitation not to consider the given 
spatial and temporal accuracy for this case. However, beyond this example, this reasoning may apply for many real-world applications, as similar or even lower accuracies may be obtained from existing tracking technologies. Further, we note that only a limited part of the lower prism intersects with the beeline connector from $o$ to $d$ (Fig. 11), which contrasts sharply with the case of an unconstrained environment emphasizing the impact of accounting for intermediate obstacles.

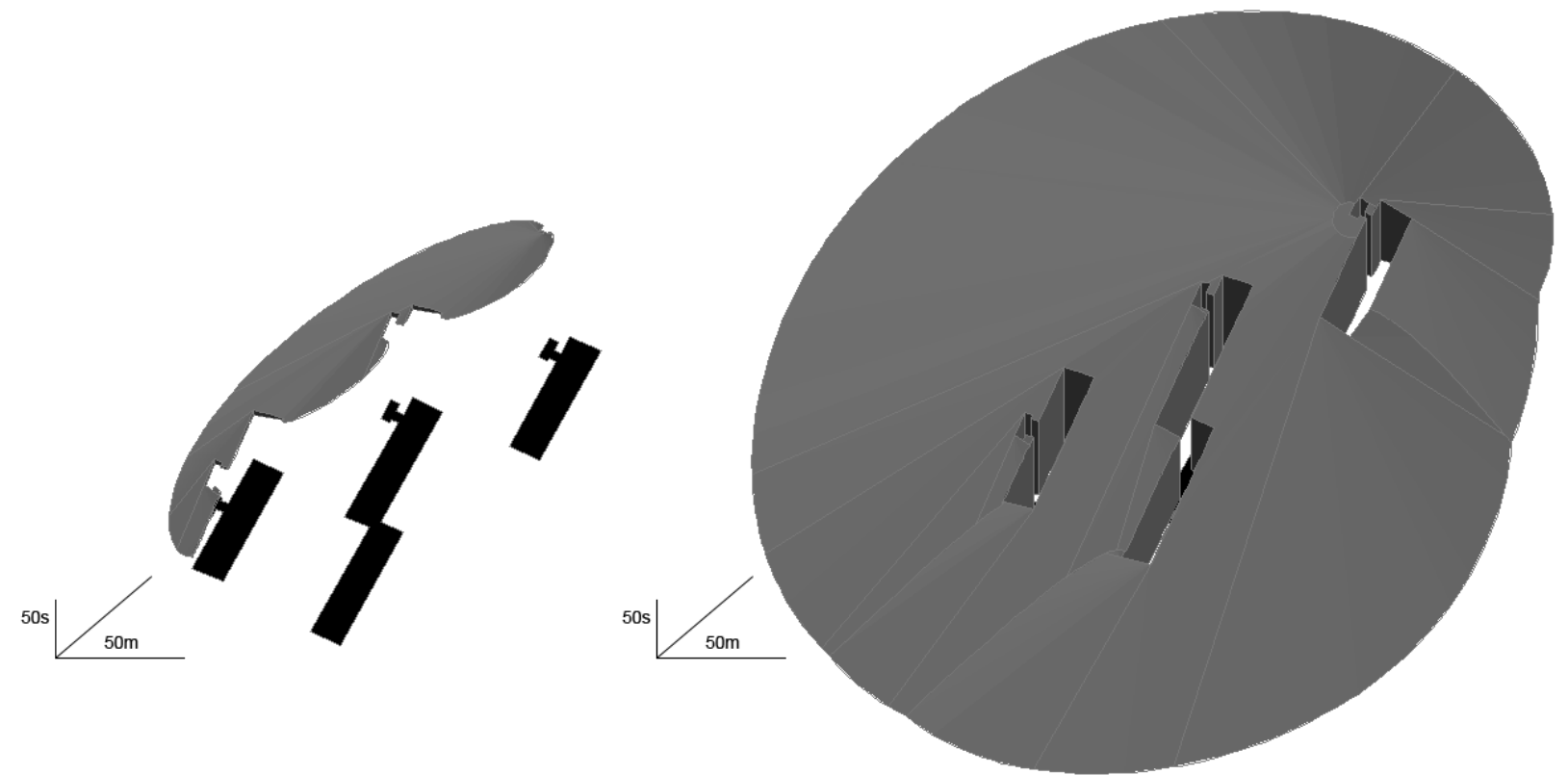

Fig. 9 Obstacle-constrained lower space-time prism

Fig. 10 Obstacle-constrained upper space-time prism

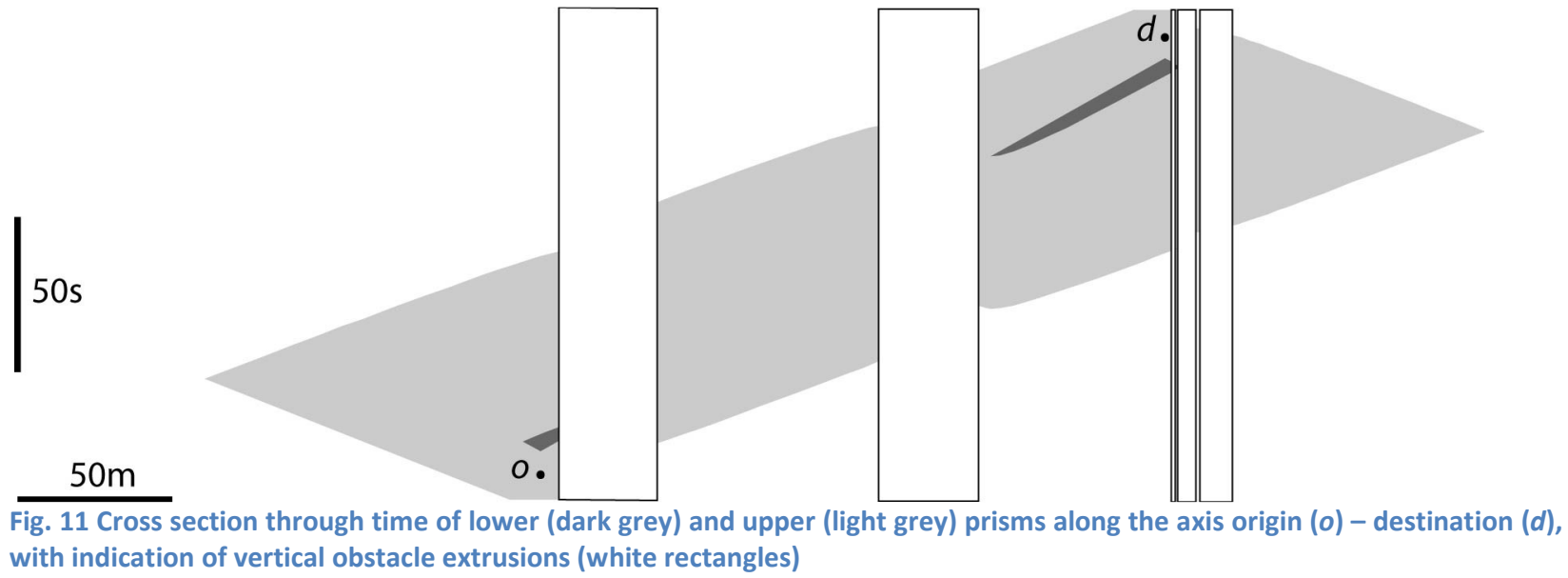

The resulting lower and upper prisms can be considered a basis for further analysis. The volume of a space-time prism, for instance, may be used as a measure of general accessibility (Lenntorp 1978, Burns 1979, Villoria 1989). Let us apply this measure in order to illustrate the impact of our approach. Table 1 presents the resulting volumes for all four scenarios that arise from taking into account or otherwise neglect the uncertainty and/or the obstacles. We obtain significantly smaller volumes when accounting for the uncertainty and for the obstacles. Ignoring uncertainty, we find a restriction to $68 \%$ when taking account of the obstacles. Analogously, when 
considering uncertainty, we achieve restrictions to $13 \%$ and $82 \%$ for lower and upper approximations respectively. Hence, with respect to the prism volume, we may conclude that, for this case, considerable overestimates are to be made whenever we neglect either the uncertain constraints, or the obstacles.

Table 1 Space-time prism volumes in $\mathrm{m}^{2}$.s according to four different scenarios

\begin{tabular}{|c|r|r|}
\hline & without uncertainty* & with uncertainty \\
\hline unconstrained & 1908020 & $\begin{array}{r}\text { (lower) 185 383 } \\
\text { (upper) 5 423 407 }\end{array}$ \\
\hline obstacle-constrained & 1297306 & $\begin{array}{r}\text { (lower) 24 125 } \\
\text { (upper) 4 432 806 }\end{array}$ \\
\hline$*$ taking $v_{\max }=\frac{v_{L}+v_{U}}{2}=2.141 \mathrm{~m}^{-1}$ & \\
\hline
\end{tabular}

\section{Conclusions}

Taking the viewpoint of nowadays tracking technologies, our contribution to time geography is twofold. First, it was shown how classical time-geographical concepts can be redefined in order to model the uncertainty associated with their underlying constraints (section 3). Typically with tracking data, uncertainties will arise from inaccuracies, errors and noise associated with the technology at hand. Relying on the basic principles of rough set theory, we have formally elaborated how space-time prisms under uncertainty can be described as rough sets with lower and upper approximations. Not only are these approximations conceptually appealing, they are also computationally efficient and robust as they allow an easy integration of different sorts of uncertainty.

Secondly, we have proposed an alternative to the assumption of unconstrained travel environment by assuming an isotropic space studded with impassable obstacles (section 4). A comprehensible methodology for the construction of space-time prisms according to this alternative assumption was elaborated. We may find many kinds of environments, both indoors and outdoors, that might be acceptably abstracted to isotropic spaces with impassable obstacles. Pedestrian precincts in urban environments, among others, are usually open, freely accessible and populated with discrete obstacles such as buildings, monuments, fenced or hedged areas, etc. Our approach complements earlier studies that have modelled space-time prisms within transportation networks. It also adds to the recent work by Miller and Bridwell (2009) who propose a fieldbased representation implemented as a lattice approximation. Although their approach allows for a complete relaxation of the uniform velocity assumption, it will be a less efficient solution, in terms of both storage and computation, in case of isotropic environments with obstacles. Our approach avoids the elongation and deviation errors related to a lattice approximation, and offers a valuable alternative if the necessary data is lacking to build a reliable and fully covering velocity field.

Both contributions, when integrated (section 5), offer a framework for time geography to represent and analyse uncertain spatiotemporal data in an environment constrained by obstacles. The yet obtained rough and obstacle-constrained space-time prisms allow for the assessment of the impact of different spatial and temporal uncertainty factors as well as various configurations 
of obstructs on accessibility. Rough obstacle-constrained prisms, and by extension the chains (or necklaces) of chronologically successive prisms, are powerful tools for accessibility analysis. The approach presented will be particularly effective for micro-scale applications because the smaller the travel environment and time budgets, the more impact spatiotemporal uncertainty will have and the less acceptable will be the ignorance of obstacles. While it may be acceptable to neglect uncertainty and abstract entire cities or urban districts as network-constrained spaces at a macro or meso scale (e.g. Kwan 1999, Weber et al. 2002, Weber 2003, Kwan et al. 2004), this reasoning may not apply when focusing on city centres and urban neighbourhoods at a micro scale. Therefore, we believe that our approach may provide increased insights into various microscale applications, including monitoring tourists or mass event visitors, crowd management, crime scene analysis, disaster management and evacuation planning.

Several extensions and refinements of our model should be addressed in future work. Since the concept of a space-time prism has now gained an acceptable degree of realism in order to analyse common tracking data in obstacle-constrained environments, we are planning to validate our methodology by means of extensive data sets. Particular emphasis will be placed on how to employ the proposed concepts to infer additional knowledge about trajectories and to measure the accessibility in space and time (Dijst et al. 2002, Shoval et al. 2007, Neutens et al. 2008, Schwanen et al. 2008, Berger et al. 2009). Further, we could consider alternatives to modelling uncertainty. Detailed and abundant numerical uncertainty data, if available, may validate the calculation of presence probabilities or membership functions. These functions, however, may significantly complicate the proposed methodology, especially when it comes to the combination of different sorts of uncertainty. Concerning the environmental constraints, an appealing extension could be to consider time-varying constraints. Instead of permanent obstacles, this would allow for handling temporary objects such as those associated to temporary events (e.g. stages, tents, and stands during a festival). Also, we might consider obstacles with passable interiors for which then different constraints apply. Lawn and bushes patches in a park, for example, could, instead of isotropic space, be considered permeable obstacles with a deviant maximum velocity with respect to pedestrian visitors.

\section{Acknowledgements}

The authors are grateful to the three anonymous reviewers for their careful reading, constructive comments and insightful suggestions. Matthias Delafontaine and Tijs Neutens acknowledge the financial support of the Research Foundation - Flanders.

\section{References}

Schiller, J. and Voisard, A., 2004, Location Based ServicesMorgan Kaufmann Publishers Inc.). Stefanidis, A. and Nittel, S., 2003, GeoSensor NetworksCRC).

Hornsby, K. and Egenhofer, M.J., 2002, Modeling moving objects over multiple granularities. Annals of Mathematics and Artificial Intelligence, 36, pp. 177-194.

Sistla, P.A., Wolfson, O., Chamberlain, S. and Dao, S., 1998, Querying the uncertain position of moving objects. In Temporal Databases: Research and Practice, O. Etzion, S. Jajodia and S. Sripada (Eds.), pp. 310-337 (Berlin: Springer). 
Moreira, J., Ribeiro, C. and Saglio, J.-M., 1999, Representation and Manipulation of Moving Points: An Extended Data Model for Location Estimation. Cartography and Geographic Information Science, 26, pp. 109-124.

Trajcevski, G., Wolfson, O., Hinrichs, K. and Chamberlain, S., 2004, Managing uncertainty in Moving Objects Databases. Acm Transactions on Database Systems, 29, pp. 463-507.

Pfoser, D., Tryfona, N. and Jensen, C., 2005, Indeterminacy and spatiotemporal data: Basic definitions and case study. Geoinformatica, 9, pp. 211-236.

Hägerstrand, T., 1970, What about people in Regional Science? Papers in Regional Science, 24, pp. 6-21.

Miller, H.J., 1991, Modelling accessibility using space-time prism concepts within geographical information systems. International Journal of Geographical Information Systems, 5, pp. 287 301.

Kwan, M.-P. and Hong, X.D., 1998, Network-based constraint-oriented choice set formation using GIS. Journal of Geographical Systems, 5, pp. 139-162.

$\mathrm{Yu}, \mathrm{H}$. and Shaw, S.L., 2008, Exploring potential human activities in physical and virtual spaces: a spatio-temporal GIS approach. International Journal of Geographical Information Science, 22, pp. 409-430.

Miller, H., J. , 2005, A Measurement Theory for Time Geography. Geographical Analysis, 37, pp. 17-45.

Neutens, T., Witlox, F., De Weghe, N.V. and De Maeyer, P., 2007a, Human interaction spaces under uncertainty. Transportation Research Record, pp. 28-35.

Pawlak, Z., 1982, Rough Sets. International Journal of Information and Computer Science, 11, pp. 341-356.

Pred, A., 1977, The Choreography of Existence: Comments on Hagerstrand's Time-Geography and Its Usefulness. Economic Geography, 53, pp. 207-221.

Lenntorp, B., 1978, A time-geographical simulation of activity programs. In Timing Space and Spacing Time, Human Activity and Time Geography, T. Carlstein, D. Parkes and N. Thrift (Eds.), pp. 162-180 (London: Edward Arnold).

Carlstein, T., Parkes, D. and Thrift, N., 1978, Timing space and spacing timeEdward Arnold).

Pred, A., 1981, Social Reproduction and the Time-Geography of Everyday Life. Geografiska Annaler. Series B, Human Geography, 63, pp. 5-22.

Yu, H. and Shaw, S.-L., 2007, Revisiting Hägerstrand's time-geographic framework for individual activities in the age of instant access. In Societies and Cities in the Age of Instant Access, pp. 103-118.

Schwanen, T. and de Jong, T., 2008, Exploring the Juggling of Responsibilities with Space-Time Accessibility Analysis. Urban Geography, 29, pp. 556-580.

Axhausen, K.W. and Gärling, T., 1992, Activity-based approaches to travel analysis: conceptual frameworks, models, and research problems. Transport Reviews: A Transnational Transdisciplinary Journal, 12, pp. 323 - 341.

Timmermans, H., Arentze, T. and Joh, C.H., 2002, Analysing space-time behaviour: new approaches to old problems. Progress in Human Geography, 26, pp. 175-190.

Dong, X.J., Ben-Akiva, M.E., Bowman, J.L. and Walker, J.L., 2006, Moving from trip-based to activity-based measures of accessibility. Transportation Research Part a-Policy and Practice, 40, pp. 163-180.

Miller, H.J. and Wu, Y.-H., 2000, GIS Software for Measuring Space-Time Accessibility in Transportation Planning and Analysis. Geoinformatica, 4, pp. 141-159. 
Wu, Y.H. and Miller, H.J., 2001, Computational tools for measuring space-time accessibility within dynamic flow transportation networks. Journal of Transportation and Statistics, 4, pp. 114.

Weber, J. and Kwan, M.-P., 2002, Bringing Time Back In: A Study on the Influence of Travel Time Variations and Facility Opening Hours on Individual Accessibility. The Professional Geographer, 54, pp. 226-240.

Kim, H.-M. and Kwan, M.-P., 2003, Space-time accessibility measures: A geocomputational algorithm with a focus on the feasible opportunity set and possible activity duration. Journal of Geographical Systems, 5, pp. 71-91.

Kuijpers, B. and Othman, W., 2009, Modeling uncertainty of moving objects on road networks via space-time prisms. International Journal of Geographical Information Science, 23, pp. 1095 1117.

Miller, H.J. and Bridwell, S.A., 2009, A Field-Based Theory for Time Geography. Annals of the Association of American Geographers, 99, pp. 49 - 75.

Puu, T. and Beckmann, M., 1999, Continuous space modelling. In Handbook of transportation science, R.W. Hall (Ed.)Kluwer Academic Publishers, Amsterdam), pp. 269-310.

Goodchild, M.F., 1977, An evaluation of lattice solutions to the problem of corridor location. Environment and Planning A, 9, pp. 727-738.

Hall, R.W., 1983, Travel outcome and performance: The effect of uncertainty on accessibility. Transportation Research Part B: Methodological, 17, pp. 275-290.

Ettema, D. and Timmermans, H., 2007, Space-time accessibility under conditions of uncertain travel times: Theory and numerical simulations. Geographical Analysis, 39, pp. 217-240.

Hendricks, M.D., Egenhofer, M.J. and Hornsby, K., 2003, Structuring a wayfinder's dynamic space-time environment. Spatial Information Theory, Proceedings, 2825, pp. 75-92.

Neutens, T., Witlox, F., Van de Weghe, N. and De Maeyer, P., 2007b, Human interaction spaces under uncertainty. Transportation Research Record, pp. 28-35.

Kapoor, S., Maheshwari, S.N. and Mitchell, J.S.B., 1997, An Efficient Algorithm for Euclidean Shortest Paths Among Polygonal Obstacles in the Plane. Discrete and Computational Geometry, 18, pp. 377-383.

Hershberger, J. and Suri, S., 1999, An Optimal Algorithm for Euclidean Shortest Paths in the Plane. SIAM J. Comput., 28, pp. 2215-2256.

Inkulu, R. and Kapoor, S., 2009, Planar rectilinear shortest path computation using corridors. Computational Geometry, 42, pp. 873-884.

Cullen, I. and Godson, V., 1975, Urban networks: the structure of activity patterns. Progress in Planning, 4, pp. 1-96.

Weber, J. and Kwan, M.P., 2003, Evaluating the effects of geographic contexts on individual accessibility: A multilevel approach. Urban Geography, 24, pp. 647-671.

Pendyala, R.M., Yamamoto, T. and Kitamura, R., 2002, On the formulation of time-space prisms to model constraints on personal activity-travel engagement. Transportation, 29, pp. 73-94.

Kitamura, R., Yamamoto, T., Susilo, Y.O. and Axhausen, K.W., 2006, How routine is a routine? An analysis of the day-to-day variability in prism vertex location. Transportation Research Part a-Policy and Practice, 40, pp. 259-279.

Shoval, N. and Isaacson, M., 2007, Sequence Alignment as a Method for Human Activity Analysis in Space and Time. Annals of the Association of American Geographers, 97, pp. 282297. 
Kapoor, S. and Maheshwari, S.N., 1988, Efficient algorithms for Euclidean shortest path and visibility problems with polygonal obstacles. In Proceedings of the fourth annual symposium on Computational geometry (Urbana-Champaign, Illinois, United States: ACM).

Mitchell, J.S.B., 1993, Shortest paths among obstacles in the plane. In Proceedings of the ninth annual symposium on Computational geometry (San Diego, California, United States: ACM).

Bohannon, R.W., 1997, Comfortable and maximum walking speed of adults aged 20-79 years: reference values and determinants. Age Ageing, 26, pp. 15-19.

Burns, L.D., 1979, Transportation, temporal, and spatial components of accessibilityLexington Books).

Villoria, O.G., 1989, An operational measure of individual accessibility for use in the study of travel-activity patterns. Ohio State University.

Kwan, M.-P., 1999, Gender and Individual Access to Urban Opportunities: A Study Using Space-Time Measures. The Professional Geographer, 51, pp. 211 - 227.

Weber, J., 2003, Individual accessibility and distance from major employment centers: An examination using space-time measures. Journal of Geographical Systems, 5, pp. 51-70.

Kwan, M., Lee, J., Michael, I., Goodchild, F. and Janelle, D.G., 2004, Geovisualization of Human Activity Patterns Using 3D GIS: A Time-Geographic Approach. Spatially Integrated Social Science, pp. 48-66.

Dijst, M., de Jong, T. and van Eck, J.R., 2002, Opportunities for transport mode change: an exploration of a disaggregated approach. Environment and Planning B: Planning and Design, 29, pp. 413-430.

Neutens, T., Schwanen, T., Witlox, F. and De Maeyer, P., 2008, My space or your space? Towards a measure of joint accessibility. Computers Environment and Urban Systems, 32, pp. 331-342.

Berger, F., Klein, R., Nussbaum, D., Sack, J.-R. and Yi, J., 2009, A meeting scheduling problem respecting time and space. Geoinformatica, 13, pp. 453-481. 